\title{
Propiedades psicométricas de una versión en castellano de la escala Motor Activity Log-30 en pacientes con extremidad superior parética por accidente cerebro vascular Psychometric properties of a Spanish version of Motor Activity Log-30 in patients with hemiparetic upper extremity due to stroke
}

Arlette Doussoulin S. ${ }^{1}$, José Luis Saiz ${ }^{2}$, Sarah Blanton ${ }^{3}$

\begin{abstract}
Background: Motor Activity $\log (M A L)$ a structured interview for stroke patients to assess the use of their paretic arm. MAL was translated and validated in Spanish. Objective: Examine the reliability and construct validity between MAL, Action Research Arm (ARA), age, pain and Stroke Impact Scale 3.0 (SIS). Methods: 40 patients from the Public Health System were evaluated. The first measurement in which clinical and socio demographic background, MAL-30 and ARA were applied. A month later; the second measurement was applied using MAL-30, ARA, and SIS 3.0. Outcome measures: The reliability was evaluated, as the internal consistency for scores of quantity and quality of movement, as well as for scores of ARA and hand function subscale SIS 3.0. The degree of agreement MAL scales, assessed through the Pearson r. Construct validity assessed by correlating scores of the first and second measurement of quantity and quality of MAL, ARA, age, pain and spasticity. As well as between the scores of the second measurement of MAL and hand function of SIS 3.0, using the Pearson $r$ and Spearman rho. Results: The quantity and quality scores of MAL-30 showed adequate levels of both internal consistency and temporal stability. In addition to an appropriate construct validity by correlating, significantly and in the expected direction, with ARA and the hand function, as well as with age, pain and spasticity. Conclusions: The evidence indicates that MAL-30 is a reliable and valid instrument to evaluate the use of the paretic arm in Spanish language patients.
\end{abstract}

Key words: Upper extremity, stroke, functional evaluation.

Rev Chil Neuro-Psiquiat 2013; 51 (3): 201-210

Recibido: 05/03/2013

Aceptado: 23/07/2013

Financiamiento: Dirección de Investigación, Universidad de La Frontera, Temuco, Chile. (Proyecto DIUFRO 09-0003).

Académico Departamento de Pediatría y Cirugía Infantil, Universidad de La Frontera, Temuco, Chile.

Académico Departamento de Psicología, Universidad de La Frontera, Temuco, Chile.

Emory Center For Rehabilitation Medicine, Emory University, Atlanta, United States of America. 


\section{Introducción}

U na de las principales causas de discapacidad y muerte a nivel mundial es el Accidente Cerebro Vascular $(\mathrm{ACV})^{1}$. En Chile, la epidemiología no es distinta; el ACV es la segunda causa específica de muerte desde hace más de 10 años, siendo responsable del 8,9\% de las defunciones y constituyendo la primera causa de hospitalizaciones sobre los 65 años ${ }^{2}$. En cuanto a demanda de atención en salud, el ACV constituye el 1,2\% de todos los egresos hospitalarios y el 4,3\% en los mayores de 65 años, considerada como la población de mayor riesgo. Por lo anterior, el ACV es considerado una prioridad de salud en Chile y, como tal, es atendido mediante el plan de Atención Universal de Garantías Explícitas $(\mathrm{AUGE})^{2}$.

Después de un año de ocurrido el ACV, un segmento relevante de los pacientes (55\%-75\%) presenta daño en la función del brazo contrario al foco de lesión cerebral ${ }^{3}$. El no uso del brazo afectado provoca un deterioro de la función, lo que limita el desarrollo de las actividades de la vida diaria (AVD), e induce una disminución en la calidad de vida y una menor productividad.

Así, la recuperación de la función de la extremidad superior parética es un objetivo primordial en el pronóstico ${ }^{4}$ y tratamiento de las secuelas neurológicas del paciente que ha sufrido un ACV. En esta perspectiva resulta primordial contar con instrumentos que evalúen de modo confiable y válido la función motora tanto de modo transversal como longitudinal.

La escala Motor Activity Log o $\mathrm{MAL}^{5-7}$ es una entrevista que evalúa la cantidad de uso (subescala $\mathrm{CU}$ ) y calidad de movimiento (subescala CM) de la mano y brazo paréticos en AVD en individuos con secuela de ACV. Esta medida se basa en el autoreporte del paciente y no una evaluación directa de la ejecución motora (Apéndice 1). Varios estudios han mostrado que diversas versiones de la escala MAL proporcionan información confiable y válida ${ }^{7-8}$.

En Chile la disponibilidad de instrumentos psicométricamente apropiados para evaluar la función motora de la extremidad superior afectada por ACV es limitada? .
A fin de contribuir a resolver este déficit, se examinaron las características psicométricas de la escala MAL-30 en pacientes chilenos con esta patología. La confiabilidad de las dos escalas MAL fue analizada según la consistencia interna de sus ítems y la estabilidad temporal de sus puntajes. Diversos estudios han mostrado que ambas subescalas de MAL son internamente consistentes, con coeficientes Alfa de Cronbach superiores a $0,80^{8} \mathrm{y}$ relativamente estable a través del tiempo, con correlaciones test-retest superiores a $0,91^{8}$.

Desde la perspectiva de la validez de constructo, se examinó en qué medida las subescalas de MAL correlacionan con cinco variables que, de acuerdo a la teoría y evidencia, deberían estar asociadas. Los antecedentes teóricos y/o empíricos que guiaron este examen son revisados a continuación.

El reporte del paciente sobre el uso espontáneo del brazo y mano paréticos en AVD debería estar asociado tanto con el uso eficiente de la extremidad como con la percepción subjetiva de tal eficiencia. Confirmando esta expectativa, estudios han encontrado que las subescalas de MAL correlacionan de modo directo tanto con la habilidad para manipular objetos ${ }^{10}$ como con la habilidad percibida en el uso de la mano afectada ${ }^{11}$.

Debido a que la funcionalidad del brazo y mano paréticos resulta disminuida por la alteración del tono muscular ${ }^{12}$, las subescalas de MAL deberían correlacionar de manera inversa con el nivel de espasticidad del paciente.

El dolor posterior al ACV es un síntoma serio $y$ frecuente que interfiere las actividades diarias ${ }^{13} y$ la rehabilitación del paciente ${ }^{14}$. Consecuentemente, debería encontrarse una relación inversa entre las subescalas de MAL y el nivel de dolor reportado. Existe evidencia que en pacientes secuelados por $\mathrm{ACV}$ el desempeño motor ${ }^{15} \mathrm{y}$ la recuperación funcional $^{16}$ disminuyen con la edad. En esta misma línea, se ha encontrado que bajos puntajes de MAL se asocian a mayor edad ${ }^{17}$. Por tanto, debería encontrarse una relación inversa entre las subescalas de MAL y la edad de los pacientes.

En consecuencia, la validez de constructo debería ser confirmada si los puntajes de MAL-30 correlacionan positivamente con a) la habilidad 
para manipular objetos; b) el uso de la extremidad afectada, y correlacionar negativamente con c) el nivel de espasticidad; d) el nivel de dolor y e) la edad del paciente.

\section{Objetivo}

Examinar las propiedades psicométricas de una versión de MAL compuesta por 30 ítems (MAL-30) traducida al castellano y adaptada a pacientes chilenos con secuela de ACV. Dos interrogantes guiaron la investigación: 1) ¿Son las subescalas CU y CM de MAL-30 internamente consistentes y estables a través del tiempo? y 2) ¿Presentan estas escalas validez de constructo en función de las cinco variables anteriormente discutidas, usadas como criterio?

\section{Pacientes y Método}

\section{Pacientes}

Una muestra no probabilística (intencionada) fue obtenida según el siguiente procedimiento. Se revisaron las fichas clínicas de 640 personas diagnosticadas con ACV en establecimientos de salud públicos y privados de la ciudad de Temuco, Chile. De éstos, se identificaron 94 individuos que cumplían con los criterios primarios de elegibilidad, tenían entre 18 y 80 años, presentaban un evento único de $\mathrm{ACV}$, y residía en zonas urbanas. Posteriormente, a partir de este segundo conjunto de pacientes se obtuvo la muestra final compuesta por 40 personas que cumplían con los criterios secundarios de elegibilidad: a) diagnóstico de ACV confirmado por Tomografía Axial Computarizada; b) evolución del ACV mayor a 6 meses; c) ausencia de daño cognitivo severo (puntaje en Escala Mini Mental $>21)^{18}$ y e) consentimiento informado escrito, aprobado previamente por la Comisión de Ética de la Dirección Regional del Servicio de Salud Araucanía Sur, Chile. En la Tabla 1 se presentan las características demográficas y clínicas relevantes de la muestra.

\section{Diseño}

Se empleó un diseño correlacional, confirmatorio y longitudinal, con dos momentos de medición (Tiempo 1 y Tiempo 2), separados por el lapso de 1 mes.

\section{Mediciones}

Siguiendo el procedimiento estándar ${ }^{19}$, se administró la escala MAL-30 a los pacientes. Además, se administraron otros seis instrumentos.

1. Action Research Arm (ARA). Es un test de desempeño que evalúa la habilidad de la extremidad superior afectada para recoger, mover y tomar objetos de diferente tamaño, peso y forma en pacientes con lesión cortical ${ }^{20-21}$. Está compuesto por 19 ítems que examinan agarre, tomada, pinza y movimiento grueso. El puntaje total va desde 0 (ninguno de los movimientos fue realizado) hasta 57 (todos los movimientos fueron realizados sin dificultad). Las propiedades psicométricas de ARA han sido bien establecidas ${ }^{9,21}$. ARA fue administrada según el protocolo descrito en el manual ${ }^{22}$. En el presente estudio este instrumento exhibió una

Tabla 1. Características demográficas y clínicas relevantes de la muestra

\begin{tabular}{|lc|}
\hline Demográficas & \\
Edad (promedio \pm desviación estándar) & $68,2 \pm 9,1$ \\
Mujeres (frecuencia) - (\%) & $20-50 \%$ \\
Clínicas & \\
Tipo de ACV (frecuencia) - (\%) & $16-40 \%$ \\
$\quad$ Hemorrágico & $24-60 \%$ \\
Isquémico & $576 \pm 237$ \\
Evolución en días (promedio \pm desviación estándar) & $1,55 \pm 1,18$ \\
Dolor (EVA) (promedio \pm desviación estándar) & $1,02 \pm 0,57$ \\
Espasticidad (EMA) (promedio \pm desviación estándar) & $24,35 \pm 3,36$ \\
Estado cognitivo (MMSE) (promedio \pm desviación estándar) & \\
\hline
\end{tabular}


adecuada consistencia interna ( $\alpha=0,88$ y 0,89 en Tiempos 1 y 2, respectivamente). Además mostró una excelente estabilidad temporal (correlación $r$ test-retest de 0,93, p $<0,001$, entre Tiempo $1 \mathrm{y}$ Tiempo 2).

2. Stroke Impact Scale versión 3.0 (SIS 3.0). Es un instrumento de autoreporte que entrega una evaluación exhaustiva de diferentes aspectos del funcionamiento diario en pacientes que han sufrido $\mathrm{ACV}^{23}$. Está compuesto por 60 preguntas agrupadas en ocho dominios. En esta investigación sólo fue analizado el dominio Función de la Mano, que evalúa la facilidad con que el paciente usa la mano afectada en las AVD. Cada dominio genera un puntaje entre 0 y 100. SIS 3.0 ha mostrado ser un instrumento confiable y válido ${ }^{24}$. En el presente estudio la consistencia interna de este dominio fue adecuada $(\alpha=0,80)$. La escala SIS 3.0 fue aplicada siguiendo el protocolo estándar ${ }^{24}$.

Al no disponer de versiones en castellano de MAL-30, ARA y SIS 3.0, estos instrumentos fueron traducidos del inglés al castellano por cuatro expertos bilingües empleando retrotraducción ${ }^{25} \mathrm{y}$ enfoque de comité ${ }^{26}$. Simultánea a la traducción, los instrumentos fueron adaptados al contexto lingüístico y cultural chileno mediante descentramiento ${ }^{27}$.

3. Escala Modificada de Ashworth (EMA). Esta escala de ítem único evalúa el tono muscular del brazo afectado, otorgando puntajes desde 0 (normal) a 4 (rígido). EMA es considerada una medida para evaluar la espasticidad, demostrando adecuados índices de confiabilidad y validez ${ }^{28}$. Se empleó una versión en castellano de esta escala ${ }^{29}$.

4. Escala Visual Análoga (EVA). Esta escala de ítem único evalúa la percepción de dolor del paciente mediante un continuo que va desde 1 (no dolor) hasta 10 (máximo dolor). EVA cuenta con evidencia que respalda su calidad psicométrica ${ }^{30}$. Se usó una versión chilena de $\mathrm{EVA}^{31}$.

5. Mini-Mental Status Examination (MMSE). Esta escala proporciona una medida breve y sen- cilla del estado cognitivo del paciente cubriendo cinco áreas: 1) orientación; 2) fijación; 3) concentración y cálculo; 4) memoria y 5) lenguaje. El puntaje total va de 0 a 30 puntos $^{18}$, con mayores puntajes indicando mayor indemnidad cognitiva. Es una prueba cuya confiabilidad y validez en castellano han sido demostradas ${ }^{32}$.

EL MMSE fue usado en este estudio sólo para propósitos de selección y/o descripción de los pacientes.

6. Ficha de antecedentes. Recolecta información sobre diversos aspectos clínicos y socio-demográficos de los pacientes. Esta información fue empleada sólo para seleccionar y/o describir la muestra.

\section{Procedimiento}

Dos ayudantes de investigación especialmente entrenadas realizaron dos mediciones separadas por un mes, sin intervención en el lapso intermedio. EMA y EVA fueron administrados en Tiempo 1 en tanto que SIS 3.0 fue administrada en tiempo 2. MAL-30 y ARA fueron administradas en ambos tiempos.

\section{Análisis estadístico}

La confiabilidad por consistencia interna de los puntajes CU y CM de MAL-30 fue evaluada a través del coeficiente alfa $(\alpha)$ de Cronbach. La confiabilidad por estabilidad temporal (test-retest) de cada subescala fue examinada mediante correlación intraclase, según el Modelo (A, k), entre los puntajes del Tiempo 1 y Tiempo $2^{33}$. Finalmente, la validez de constructo fue analizada a través de correlaciones simples entre, por un lado, los puntajes $\mathrm{CU}$ y CM y, por otro, puntajes de las cinco variables empleadas como criterio. Dependiendo del nivel intervalar u ordinal de medición de los criterios, en el cálculo de las correlaciones se utilizaron, respectivamente, los coeficientes $r$ de Pearson y rho de Spearman.

\section{Resultados}

En el Tiempo 1, los valores $\alpha$ fueron 0,96 tanto para los puntajes CU como CM, en tanto que en el 
Tabla 2. Resultados del examen de la validez de constructo de MAL-30

\begin{tabular}{|c|c|c|c|c|}
\hline \multirow[b]{2}{*}{ Variables criterio } & \multicolumn{2}{|c|}{$\mathrm{CM}$} & \multicolumn{2}{|c|}{$\mathrm{CU}$} \\
\hline & Tiempo 1 & Tiempo 2 & Tiempo 1 & Tiempo 2 \\
\hline $\mathrm{ARA}^{\mathrm{a}}$ (Tiempo 1) & 0,67 & -- & 0,66 & -- \\
\hline ARA $^{a}$ (Tiempo 2) & -- & 0,53 & -- & 0,59 \\
\hline Función de la mano (SIS 3.0)a & -- & 0,53 & -- & 0,62 \\
\hline $\mathrm{EMA}^{\mathrm{b}}$ & $-0,62$ & -- & $-0,64$ & -- \\
\hline $\mathrm{EVA}^{\mathrm{b}}$ & $-0,37$ & -- & $-0,48$ & -- \\
\hline Edad $^{\mathrm{a}}$ & $-0,53$ & -- & $-0,58$ & -- \\
\hline
\end{tabular}

${ }^{a}$ Correlación $r$ de Pearson. ${ }^{\mathrm{b} C o r r e l a c i o ́ n ~} r$ ho de Spearman. Nota. Todas las correlaciones son significativas con $\mathrm{p}<0,02$.

Tiempo 2, los valores $\alpha$ fueron 0,97 para CU y 0,96 para CM. Estos resultados apoyan la confiabilidad por consistencia interna de ambas subescalas de MAL-30. Las correlaciones intraclase entre Tiempo 1 y Tiempo 2 fueron 0,86 para CU y 0,81 para CM, ps $<0,001$, indicando que los puntajes de ambas subescalas se mantienen bastante estables, al menos, en el lapso de 1 mes.

En la Tabla 2 se exponen los resultados sobre la validez de constructo de MAL-30. Todas las correlaciones entre cada subescala de MAL-30 y los cinco criterios fueron significativas y en la dirección esperada. Específicamente, se obtuvieron asociaciones directas de $\mathrm{CU}$ y CM con la habilidad para manipular objetos (medida por ARA) en los Tiempos 1 y 2, como también con la facilidad de uso de la mano afectada (medida por Función de la Mano, SIS 3.0) en el Tiempo 2. Además, se obtuvieron asociaciones inversas de CU y CM con espasticidad (medida por EMA), dolor (medida por EVA) y edad. Este conjunto de resultados aportan evidencia a favor de la validez de constructo de las subescalas de MAL-30.

\section{Discusión}

Esta investigación tuvo como propósito examinar si esta versión en castellano de MAL-30 es un instrumento confiable y válido para evaluar la extremidad superior de pacientes con ACV. Los resultados obtenidos respaldan la calidad psicométrica de la MAL-30, sugiriendo que este instrumento es una medida cultural y lingüísticamente apropiada para pacientes de esta nacionalidad.

La consistencia interna mostrada por esta versión de MAL-30 indica que sus ítems miden de modo coherente un mismo constructo. Estos resultados pueden ser considerados como excelentes ${ }^{33}$, siendo incluso superiores a los reportados por estudios previos en pacientes de otras nacionalidades ${ }^{7-8}$. Por otra parte, el alto grado de acuerdo test-retest obtenido señala que MAL-30 proporciona puntajes que se mantienen estables a través del tiempo, en ausencia de intervención, coincidiendo con van de Lee, $2004^{7}$.

Al examinar la validez de constructo de MAL30 se observa que sus puntajes se asocian efectivamente con otras variables del paciente del modo teóricamente y/o empíricamente esperado, aportando evidencia de que este instrumento parece medir adecuadamente la cantidad y calidad de uso del brazo y mano paréticos durante actividades cotidianas en pacientes con ACV. Es interesante notar que esta validez se apoya en correlaciones significativas de MAL-30 tanto con medidas directas de ejecución (ARA y EMA) como con medidas autoreportadas por el paciente (Función de la Mano y EVA). Aún cuando las medidas autoreportadas pueden estar más expuestas a error y, por consiguiente, a una merma en su confiabilidad, la convergencia de resultados con estos dos tipos de medidas parece reforzar la validez de MAL-30.

La asociación directa entre MAL-30 y ARA indica que el reporte subjetivo del uso de la extremidad afectada tiende a ser consistente con el desempeño 
real del paciente con esa extremidad, específicamente con la habilidad de manipular objetos ${ }^{10}$. La correlación, también directa, entre MAL-30 y Función de la Mano señala que mientras mayor es la cantidad de uso y calidad de movimiento de la extremidad superior, mayor es la habilidad percibida del paciente en el empleo de la mano afectada ${ }^{11}$.

Por otra parte, se obtuvo una correlación inversa entre MAL-30 y EMA, que evalúa el tono muscular. Esta asociación parece fundarse en el hecho que mientras mayor es la alteración del tono muscular, expresado en espasticidad por el daño de primera motoneurona, menor es el uso de la mano y brazo parético del sujeto en sus actividades cotidianas ${ }^{12}$.

Así también, las escalas de MAL-30 correlacionaron de modo inverso con EVA, que evalúa percepción de dolor en la extremidad parética. Así, bajos niveles de cantidad y calidad de movimiento se asocian a altos niveles de dolor. Este hallazgo se fundamenta en que el dolor posterior al ACV es un síntoma serio y frecuente que dificulta y limita las actividades diarias ${ }^{13}$ y la rehabilitación del paciente $^{34}$.

Igualmente, MAL-30 y edad se asociaron de modo inverso. De este modo, cantidad de uso y calidad de movimiento disminuidos tienden a estar más presentes en los pacientes más añosos con secuela de $\mathrm{ACV}^{35}$. Consecuentemente, las personas más jóvenes tienen un mayor potencial de recuperación ${ }^{35}$.

La literatura reporta distintas versiones de MAL según la cantidad de ítems que incluyen: 14, 20, 26 ó 30 ítems ${ }^{5,36-38}$. Aparentemente, la longitud de MAL no parece afectar crucialmente sus propiedades psicométricas. Estas distintas versiones suelen proporcionar resultados muy similares entre sí, aparentemente debido a la alta consistencia interna de sus ítems ${ }^{8}$. De hecho, los resultados del presente estudio son coincidentes con los reportados previamente con versiones de diferente longitud.

Un resultado habitualmente reportado en la literatura es la elevada correlación entre las dos subescalas de $\mathrm{MAL}^{39}$. De hecho, en el presente estudio esta correlación fue $\mathrm{r}=0,97$ en el Tiempo 1 y r $=0,94$ en el Tiempo 2, ps $<0,001$. Esta alta asociación, cercana a la correlación perfecta, puede indicar redundancia entre las respectivas mediciones de las dos subescalas, haciendo aconsejable emplear una sola de ellas ${ }^{39}$, con el consiguiente ahorro de tiempo de aplicación y de recursos humanos.

La evidencia mostrada en el estudio sobre la adecuación psicométrica de MAL-30 en pacientes chilenos con ACV es concordante con la utilidad asignada internacionalmente a este instrumento como medida de resultados en ensayos clínicos. En tal se sentido, se ha demostrado que este instrumento parece ser comparativamente más idóneo para evaluar la mejora en la funcionalidad de la mano y brazo paréticos ${ }^{40}$.

Puesto que la validación de un instrumento de medición es un proceso acumulativo de evidencia, indudablemente se requieren más estudios sobre las propiedades psicométricas de MAL-30 en otras poblaciones con $\mathrm{ACV}$ o trastornos similares y/o empleando otras variables como criterio de validación. Con todo, la evidencia aquí encontrada sobre la calidad psicométrica de esta versión en castellano de MAL-30 insinúa que el empleo de este instrumento en otros países de habla castellana es promisorio, al menos, en un primer acercamiento, para propósitos de investigación y, luego, una vez demostrada su calidad psicométrica, para uso clínico. Es deseable que los resultados del presente estudio estimulen el empleo de MAL-30 en la práctica clínica de los profesionales chilenos, beneficiándose así de las ventajas de contar con una evaluación estandarizada, adaptada a la particularidades culturales y lingüísticas nacionales, y con un adecuado respaldo psicométrico inicial.

\section{Conclusiones}

Los resultados indican que las subescala de MAL-30 constituyen medidas confiables y válidas del uso, en cantidad y calidad, de la extremidad parética a causa de un ACV, en pacientes chilenos. 
Apéndice 1. Motor Activity Log (MAL)

Nombre:
Mano dominante:
Lado débil:

\begin{tabular}{|c|c|c|c|c|}
\hline & Registro de la actividad motora & $\begin{array}{l}\text { Cantidad } \\
\text { de uso }\end{array}$ & $\begin{array}{l}\text { Calidad de } \\
\text { movimiento }\end{array}$ & $\begin{array}{c}\text { Comentarios } \\
\text { Sí o no, indicar } \\
\text { por qué (códigos) }\end{array}$ \\
\hline 1 & Encender la luz con un interruptor & & & \\
\hline 2 & Abrir una cajonera & & & \\
\hline 3 & Sacar una prenda de ropa desde la cajonera & & & \\
\hline 4 & Tomar el teléfono & & & \\
\hline 5 & Limpiar con un paño una superficie & & & \\
\hline 6 & $\begin{array}{l}\text { Salir de un auto (movimiento para conseguir que el cuerpo se desplace } \\
\text { desde sentando a de pie fuera del auto, con la puerta abierta) }\end{array}$ & & & \\
\hline 7 & Abrir un refrigerador & & & \\
\hline 8 & Abrir la puerta girando una manilla & & & \\
\hline 9 & Usar el control remoto de un TV & & & \\
\hline 10 & Lavarse las manos (incluye aplicarse jabón, no incluye abrir las llaves) & & & \\
\hline 11 & Abrir y cerrar la llave del agua & & & \\
\hline 12 & Secar sus manos & & & \\
\hline 13 & Ponerse calcetines & & & \\
\hline 14 & Sacarse los calcetines & & & \\
\hline 15 & Ponerse los zapatos (incluye amarrarse los cordones) & & & \\
\hline 16 & Quitarse los zapatos (incluye desamarrar los cordones) & & & \\
\hline 17 & Levantarse de una silla con apoya brazos & & & \\
\hline 18 & Tirar la silla fuera de la mesa para sentarse & & & \\
\hline 19 & Empujar una silla hacia la mesa después de sentarse & & & \\
\hline 20 & $\begin{array}{l}\text { Tomar un vaso o botella o taza para beber (no es necesario beber, solo } \\
\text { llevarla a la boca) }\end{array}$ & & & \\
\hline 21 & Cepillarse los dientes (no incluye aplicar la pasta) & & & \\
\hline 22 & Aplicarse maquillaje o loción o crema de afeitar & & & \\
\hline 23 & Usar una llave para abrir la puerta & & & \\
\hline 24 & Escribir sobre un papel & & & \\
\hline 25 & Llevar un objeto en la mano & & & \\
\hline 26 & Usar tenedor o cuchara para comer & & & \\
\hline 27 & Peinar su cabello & & & \\
\hline 28 & Tomar una taza desde el asa & & & \\
\hline 29 & Abotonar una camisa & & & \\
\hline \multirow[t]{3}{*}{30} & Comer la mitad de un pan o sándwich & & & \\
\hline & Puntaje total & & & \\
\hline & Puntaje promedio & & & \\
\hline
\end{tabular}

Códigos para responder respuestas "no".

1. "Yo utilizo solamente mi brazo indemne, para esta actividad" (asignar 0).

2. "Alguien más lo hace por mí" (asignar 0).

3. "Yo nunca hago esta actividad, con o sin ayuda de alguien más, porque es imposible". Por ejemplo peinarse, si es calvo. (asignar "N/A y sacar desde la lista de ítems).

4. "Yo algunas veces hago esta actividad, pero no he tenido la oportunidad de realizarlo desde la última vez que me realizaron la pregunta". Incluir el puntaje asignado la última vez.

5. Esta es una actividad que yo normalmente realizaba sólo con mi mano dominante antes del ACV (sólo aplicable al \# 24 y sacar desde la lista de ítems). 


\begin{abstract}
Resumen
Introducción: Motor Activity Log (MAL) es una entrevista estructurada, que evalúa el uso del brazo parético en pacientes con Accidente Cerebro Vascular. MAL fue traducida al español y validada. Objetivo: Examinar la confiabilidad y validez de constructo entre MAL, Action Research Arm (ARA), edad, dolor y la escala Stroke Impact Scale 3.0 (SIS). Método: Fueron evaluados 40 pacientes del sistema público de salud. En la primera medición se aplicó una ficha clínica y demográfica, MAL-30 y ARA. Un mes después; en la segunda medición se aplicó MAL-30, ARA y SIS 3.0. Mediciones: la confiabilidad fue evaluada a través de la consistencia interna de los puntajes de cantidad y calidad de MAL-30, los puntajes de ARA y la subescala función de la mano de SIS 3.0. El grado de acuerdo entre las escalas de MAL se evaluó a través de $r$ Pearson. La validez de constructo fue evaluada por correlación entre los puntajes de la primera y segunda medición de cantidad y calidad de MAL, ARA, edad, dolor y espasticidad. Así también, entre los puntajes de la segunda medición de MAL y función de la mano utilizando r Pearson y rho de Spearman. Resultados: MAL-30 mostró adecuada consistencia interna y estabilidad temporal. Además, de una apropiada validez de constructo correlacionando significativamente y en la dirección esperada con ARA, función de la mano, edad, dolor y espasticidad. Conclusiones: la evidencia indica que MAL-30 es un instrumento confiable y válido para evaluar el uso del brazo parético en pacientes de habla hispana.
\end{abstract}

Palabras clave: Extremidad superior, ACV, evaluación funcional.

\section{Referencias bibliográficas}

1. López A, Mathers C, Ezzati M, Jamison D, Murray C. Global and regional burden of disease and risk factors, 2001: systematic analysis of population health data. Lancet 2006; 367 (9524): 1747-57.

2. http://www.minsal.cl. Guía Clínica Ataque Cerebrovascular Isquémico del Adulto. No 37: Ministerio de Salud-República de Chile. 2007.

3. Nichols-Larsen D, Clark P, Zeringue A, Greenspan A, Blanton S. Factors influencing stroke survivors' quality of life during subacute recovery. Stroke 2005; 36: 1480-4.

4. Nijland R, Van Wegen E, Harmeling-Van Der Wel B, Kwakkel G. Presence of finger extension and shoulder abduction within 72 hours after stroke predicts functional recovery: early prediction of functional outcome after stroke: the EPOS cohort study. Stroke 2010; 41 (4): 745-50.

5. Taub E, Miller N, Novack T, Cook E, Fleming $\mathrm{W}$, Nepomuceno C, et al. Technique to improve chronic motor deficit after stroke. Arch Phys Med
Rehabil 1993; 74 (4): 347-54.

6. Uswatte G, Taub E, Morris D, Vignolo M, Mcculloch K. Reliability and validity of the upperextremity Motor Activity Log-14 for measuring real-world arm use. Stroke 2005; 36 (11): 2493-6.

7. Van Der Lee J, Beckerman H, Knol D, De Vet H, Bouter L. Clinimetric properties of the motor activity log for the assessment of arm use in hemiparetic patients. Stroke 2004; 35 (6): 1410-4.

8. Uswatte G, Taub E, Morris D, Light K, Thompson PA. The Motor Activity Log-28: assessing daily use of the hemiparetic arm after stroke. Neurology 2006; 67 (7): 1189-94.

9. Doussoulin A, Rivas R, Campos V. Validación de "Action Research Arm Test" (ARAT) en pacientes con extremidad superior parética post ataque cerebro vascular en Chile. Rev Med Chile 2012; 140: 59-65.

10. Van Der Lee J, Roorda L, Beckerman H, Lankhorst G, Bouter L. Improving the Action Research Arm test: a unidimensional hierarchical scale. Clin Rehabil 2002; 16 (6): 646-53. 
11. Feys H, De Weerdt W, Selz B, Cox Steck G, Spichiger R, Vereeck L, et al. Effect of a therapeutic intervention for the hemiplegic upper limb in the acute phase after stroke: a single-blind, randomized, controlled multicenter trial. Stroke 1998; 29 (4): 785-92.

12. Malhotra S, Pandyan A, Rosewilliam S, Roffe C, Hermens H. Spasticity and contractures at the wrist after stroke: time course of development and their association with functional recovery of the upper limb. Clin Rehabil 2011; 25 (2): 184-91.

13. Glader E, Stegmayr R, Asplund K. Poststroke fatigue: a 2 year follow-up study of stroke patient in Sweden. Stroke 2003; 33: 1327-33.

14. Ploughman M, Corbett D. Can forced-use therapy be clinically applied after stroke? An exploratory randomized controlled trial. Arch Phys Med Rehabil 2004; 85 (9): 1417-23.

15. Bagg S, Pombo A, Hopman W. Effect of age on functional outcomes after stroke rehabilitation. Stroke 2002; 33 (1): 179-85.

16. Jongbloed L. Prediction of function after stroke: a critical review. Stroke 1986; 17 (4): 765-76.

17. Fritz S, Light K, Clifford S, Patterson T, Behrman A, Davis S. Descriptive characteristics as potential predictors of outcomes following constraintinduced movement therapy for people after stroke. Phys Ther 2006; 86 (6): 825-32.

18. Folstein M, Folstein S, Mchugh P. "Mini-mental state". A practical method for grading the cognitive state of patients for the clinician. J Psychiatr Res 1975; 12 (3): 189-98.

19. Bowman M, Taub E, Uswatte G, Delgado A, Bryson $\mathrm{C}$, Morris $\mathrm{D}$, et al. A treatment for a chronic stroke patient with a plegic hand combining CI therapy with conventional rehabilitation procedures: case report. Neuro Rehabilitation 2006; 21 (2): 167-76.

20. Lyle R. A performance test for assessment of upper limb function in physical rehabilitation treatment and research. Int J Rehabil Res 1981; 4 (4): 483-92.

21. Koh C, Hsueh I, Wang W, Sheu C, Yu T, Wang C, et al. Validation of the action research arm test using item response theory in patients after stroke. J Rehabil Med 2006; 38 (6): 375-80.

22. Yozbatiran N, Der-Yeghiaian L, Cramer S. A standardized approach to performing the action research arm test. Neurorehabil Neural Repair 2009; 22 (1): 78-90.

23. Lin K, Chung H, Wu C, Liu H, Hsieh Y, Chen I, et al. Constraint-induced therapy versus control intervention in patients with stroke: a functional magnetic resonance imaging study. Am J Phys Med Rehabil 2010; 89 (3): 177-85.

24. Duncan P, Wallace D, Lai S, Johnson D, Embretson S, Laster L. The stroke impact scale version 2.0 Evaluation of reliability, validity, and sensitivity to change. Stroke 1999; 30 (10): 2131-40.

25. The wording and translation of research instruments. In: Field methods in cross-cultural psychology. Newbury Park, CA: Sage, 1986: 137-64.

26. Brislin R. Traslation and content analysis of oral and written material. In: Triandis editor. Handbook of cross-cultural psychology. Boston: Allyn and Bacon, 1980: 389-444.

27. Werner D. Translating working through interpreters, and the problem of decentering. In: Narroll R., editor. A handbook of method in cultural anthropology. Nueva York: American Museum of Natural History Press 1970: 398-420.

28. Waninge A, Rook R, Dijkhuizen a, gielen e, van der schans C. Feasibility, test-retest reliability, and interrater reliability of the Modified Ashworth Scale and Modified Tardieu Scale in persons with profound intellectual and multiple disabilities. Res Dev Disabil 2011; 32 (2): 613-20.

29. Agredo C, Bedoya J. Validación Escala de Ashworth Modificada. efisioterpia.net, 2005.

30. Lazaro C, Caseras X, Torrubia R, Banos J. Measurement of postoperative pain: analysis of the sensitivity of various self-evaluation instruments. Rev Esp Anestesiol Reanim 2003; 50 (5): 230-6.

31. Riedemann P. Clinimetría: Aspectos Generales sobre medición en enfermedades musculoesqueléticas. Revista Chilena de Reumatología 2001; 17: 173-8.

32. Giménez-Roldán M, Navarro E, Dobato J, Giménez-Zuccarelli M. Examen del estado MiniMental: propuesta de una normativa para su aplicación. Rev Neurol 1997; 25 (140): 576-83.

33. Streiner D. Starting at the beginning: an introduction to coefficient alpha and internal consistency. J 
Pers Assess 2003; 80 (1): 99-103.

34. Snels I, Beckerman H, Lankhorst G, Bouter L. Treatment of hemiplegic shoulder pain in the Netherlands: results of a national survey. Clin Rehabil 2000; 14 (1): 20-7.

35. Ward N, Frackowiak R. Age-related changes in the neural correlates of motor performance. Brain 2003; 126 (Pt 4): 873-88.

36. Van Der Lee J, Wagenaar R, Lankhorst G, Vogelaar T, Deville W, Bouter L. Forced use of the upper extremity in chronic stroke patients: results from a single-blind randomized clinical trial. Stroke 1999; 30 (11): 2369-75.

37. Liepert J, Miltner W, Bauder H, Sommer M, Dettmers C, Taub E, et al. Motor cortex plasticity during constraint-induced movement therapy in stroke patients. Neurosci Lett 1998; 250 (1): 5-8.

38. Blanton S, Wolf S. An application of upper-extremity constraint-induced movement therapy in a patient with subacute stroke. Phys Ther 1999; 79 (9): 847-53.

39. Park SW, Wolf S, Blanton S, Winstein C, NicholsLarsen D. The EXCITE Trial: Predicting a clinically meaningful motor activity log outcome. Neurorehabil Neural Repair 2008; 22 (5): 486-93.

40. Taub E, Uswatte G, King D, Morris D, Crago J, Chatterjee A. A placebo-controlled trial of constraint-induced movement therapy for upper extremity after stroke. Stroke 2006; 37 (4): 10459.
Correspondencia:

Arlette Doussoulin Sanhueza

Francisco Salazar 01145 Temuco, Chile.

Teléfono: 56-45-732411

Fax: 56-45-744308

E-mail: arlette.doussoulin@ufrontera.cl 